\title{
Vitreopapillary traction in proliferative diabetic vitreoretinopathy
}

\author{
Peter Kroll, Wolfgang Wiegand, Joerg Schmidt
}

\begin{abstract}
Aim-To present the clinical profile of a new entity in advanced proliferative diabetic vitreoretinopathy (PDVR). Mechanisms of vision loss due to vitreopapillary traction on the nasal optic disc are described, followed by an introduction of methods for prevention and treatment in such cases.

Methods-17 patients with PDVR and traction on the nasal side of the optic disc, pallor of the optic nerve head, and reduced visual acuity were included in the study. Six patients were observed retrospectively and 11 patients prospectively before and after pars plana vitrectomy. Pre- and postoperative examinations included visual acuity, Goldmann's visual field, fluorescein angiography, and measurements of visual evoked potentials (VEP).

Results-During a postoperative follow up period of 3 to 24.5 months (mean 14.5 months) an improvement in optic disc appearance combined with an increased visual acuity (mean increase in VA= 0.171 ) was observed in $15 / 17(88.3 \%)$ patients. In addition, $8 / 17(47 \%)$ of these patients showed higher VEP amplitudes (mean $3.83 \mu \mathrm{V}$ ), and eight (6/8 of the same patients as VEP amplitudes) patients
\end{abstract}

showed a reduction of latency (mean reduction $22.25 \mathrm{~ms}$ ) during VEP assessment.

Conclusion-These results suggest that vitreopapillary traction may damage the anterior optic nerve, via decreased axoplasmatic flow in the optic nerve fibres and/or mechanical reduction of perfusion in the posterior ciliary arteries. The effects of each mechanism appear to be reversible, but in the long term might lead to irreversible optic nerve atrophy. Therefore, in patients with vitreopapillary traction, early vitrectomy should be considered as a method to prevent optic neuropathy.

(Br f Ophthalmol 1999;83:261-264)

Optic nerve atrophy may be due to intraorbital or CNS compression or ischaemia producing direct effects on the optic nerve, or progressive damage to retinal ganglion cells caused by inflammation, degeneration, elevated intraocular pressure, or ischaemia. ${ }^{1-3}$ In patients with proliferative diabetic vitreoretinopathy (PDVR), another possible mechanism for nerve atrophy may be vitreopapillary traction of the optic disc, as described by De Bustros and co-workers in 1987. ${ }^{4}$ The concept of vitreopapillary traction and its relief during vitrectomy for PDVR has been debated over the past
Department of Ophthalmology, Philipps-University Marburg, Marburg, Germany P Kroll

W Wiegand

J Schmidt

Correspondence to: Professor Peter Kroll, Department of

Ophthalmology,

Philipps-University Marburg,

Robert Koch Strasse 4

35037 Marburg, Germany.

Accepted for publication 30 July 1998
Table 1 Overview of patients investigated in this study. Pre-and postoperative visual acuity was measured in all patients. Available VEP results are shown. Evaluation mode, duration of symptoms, and determination of diabetic stage are displayed for every individual

\begin{tabular}{|c|c|c|c|c|c|c|}
\hline Patient No & $\begin{array}{l}\text { Visual acuity } \\
\text { pre/post }\end{array}$ & $\begin{array}{l}\text { VEP latency (ms) } \\
\text { pre/postop }\end{array}$ & $\begin{array}{l}\text { VEP amplitude } \\
(\mu V) \text { pre/postop }\end{array}$ & $\begin{array}{l}\text { Duration of traction } \\
\text { (years) }\end{array}$ & Evaluation type & Diabetes type \\
\hline 1 & $0.02 / 0.1$ & $84 / 88$ & $8.6 / 6.6$ & 1 & prospective & II \\
\hline 2 & $0.3 / 0.5$ & $168 / 130$ & $3.7 / 3.9$ & 1 & prospective & II \\
\hline 3 & $0.02 / 0.1$ & $86 / 82$ & $3.9 / 2.9$ & 5 & prospective & II \\
\hline 4 & $0.02 / 0.05$ & $85 / 78$ & $2.8 / 14.8$ & 1 & prospective & II \\
\hline 5 & $0.5 / 0.5$ & $154 / 152$ & $4.3 / 6.4$ & 1 & prospective & I \\
\hline 6 & $0.05 / 0.1$ & $118 / 230$ & $2.3 / 12.1$ & 1.5 & prospective & II \\
\hline 7 & $0.02 / 0.4$ & $142 / 118$ & $4.5 / 3.3$ & 0.5 & prospective & II \\
\hline 8 & $0.02 / 0.2$ & NA/142 & $\mathrm{NA} / 3.9$ & 2 & prospective & II \\
\hline 9 & $0.08 / 0.02$ & NA/138 & $\mathrm{NA} / 3.9$ & 10 & retrospective & I \\
\hline 10 & $0.4 / 0.5$ & NA & NA & 1 & retrospective & II \\
\hline 11 & $0.2 / 0.3$ & NA & NA & 4 & retrospective & II \\
\hline 12 & $0.02 / 0.5$ & NA & NA & 2 & retrospective & I \\
\hline 13 & $0.3 / 0.4$ & NA & NA & 1.5 & retrospective & I \\
\hline 14 & $0.2 / 0.4$ & $130 / 80$ & $5.1 / 5.25$ & 2 & prospective & II \\
\hline 15 & $0.1 / 0.3$ & $124 / 124$ & $4.7 / 6.0$ & 3.5 & prospective & I \\
\hline 16 & $0.05 / 0.3$ & $152 / 106$ & $2.3 / 4.5$ & 6 & prospective & I \\
\hline 17 & $0.02 / 0.4$ & $142 / 135$ & $3.9 / 5.8$ & 2.5 & prospective & II \\
\hline
\end{tabular}

$\mathrm{NA}=$ data not available. 

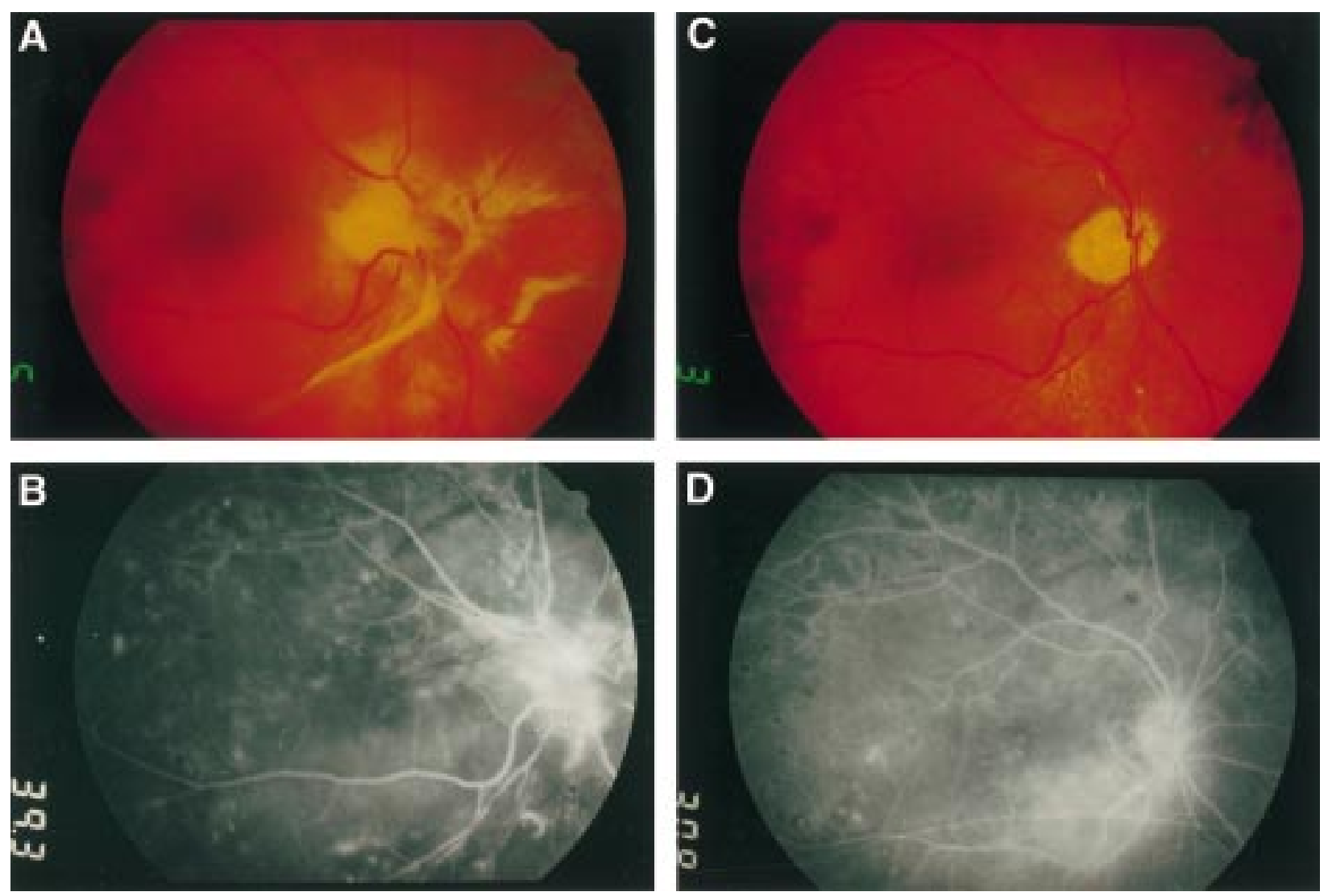

Figure 1 Diabetic patient with severe nasal vitreopapillary traction before $(A)$ and after (C) vitrectomy for removal of membranes. In (B) and (D) the corresponding fluorescein angiograms for fundus photos $(A)$ and $(C)$ are shown, respectively. In the preoperative state vitreopapillary traction is accompanied by leakage from the papillary region. The leakage resolved once traction is alleviated by membrane removal as shown in (D).

decade by a number of groups. ${ }^{4-7}$ To determine whether there is any relation between disc pallor (in combination with reduced visual acuity) and vitreopapillary traction clinical, angiographic, and electrophysiological studies were performed before and after vitrectomy in patients with PDVR. None of these patients had other identifiable causes of visual impairment such as macular oedema, vitreoretinal traction close to the macula, or severe vitreous haemorrhage.

\section{Patients and methods}

During a period of 2 years, 17 patients with PDVR and isolated vitreopapillary traction on the nasal aspect of the optic nerve head were observed (six patients retrospectively, 11 patients prospectively). All patients had reduced visual acuity and optic disc pallor. Exclusion criteria were macular oedema, vitreoretinal traction close to the macula, tractional heterotopia, and severe vitreous haemorrhage. Fluorescein angiography was performed to exclude any leakage or extensive hypoperfusion of the macular region. All patients had vitreopapillary traction ranging from 1 to 10 years in duration in one eye while the fellow eye exhibited diabetic vitreoretinopathy stage $\mathrm{A}^{7}$ with a normal optic disc, flat retina and a neovascular, fibrotic proliferation at the posterior vitreous cortex. The ages of the patients ranged from 28 to 72 years (mean 52.6 years). Six patients were female, 11 patients were male. Duration of diabetes before surgery was 4-25 years (mean 12.6 years), with six patients having type I and 11 patients type II diabetes. All patients underwent pars plana vitrectomy on the affected eye, using a three port vitrectomy system for complete removal of the vitreous. Visual acuity, Goldmann visual field, fluorescein angiography, and visual evoked potentials (VEP) were determined pre- and postoperatively by independent observers. In eyes with visual acuity better than $20 / 200$ before surgery, we obtained pattern visual evoked potentials (PVEP), in eyes with visual acuity of $20 / 200$ or

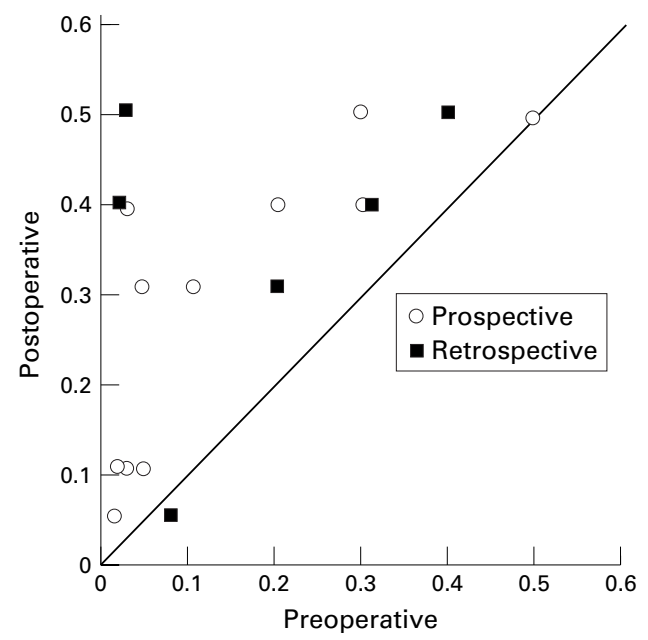

Figure 2 Scattergram of visual acuity in eyes with diabetic vitreopapillary traction before and 2 months after vitreoretinal surgery: in 15 of 17 eyes visual acuity improved postoperatively. 
Figure 3 Visual evoked potentials (VEP) in diabetic vitreopapillary traction. VEP amplitudes $(A)$ and VEP latency (B) before and 2 months after vitreoretinal surgery: in eight of 11 patients amplitudes improved after vitrectomy; in 10 of 11 patients a postoperative reduction of latency was observed.
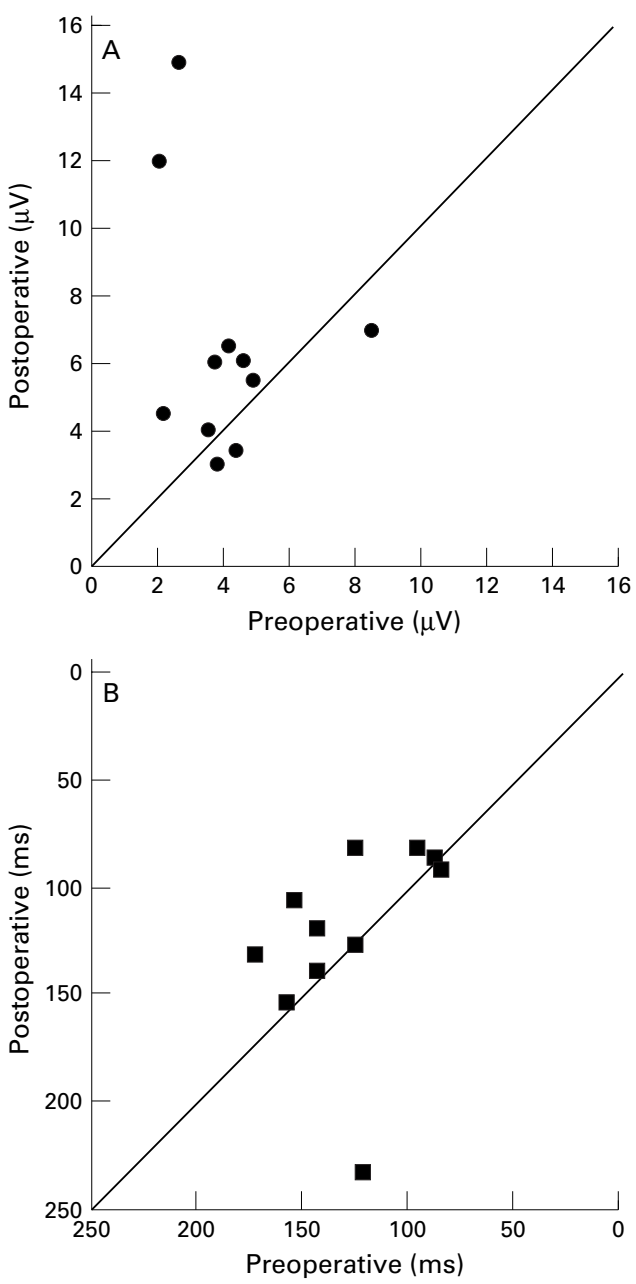

worse we performed flash visual evoked potentials (FVEP). The postoperative follow up lasted between 3 and 24.5 months (mean 14.5 months).

\section{Results}

Vitreous attachments to the optic disc could be removed without complications in all patients (Table 1). It was our subjective impression that the preoperatively pale optic disc regained a better colour (Fig 1C) in all but one case. However, no further evaluation was performed because of the subjectivity of this observation. Fluorescein angiography (examples: Fig 1B and D) before vitrectomy showed typical neovascularisation of the optic disc with leakage and the absence of any leakage or extensive non-perfusion of the macular region. Leakage disappeared after removal of the posterior vitreous cortex and relief of vitreopapillary traction in all cases (examples: Fig 1B and D). In 15 of 17 eyes (88.3\%) visual acuity improved postoperatively by a mean of 0.174 Snellen units, or $9.7 \%$ above preoperative levels (Fig 2). Of the two patients who had no improvement in visual acuity after surgery, one had vitreopapillary traction persisting for 1 year with a preoperative visual acuity of $20 / 40$, which remained unchanged after vitrectomy. Even this eye, however, gained a marked improvement in VEP amplitudes $(4.3-6.4 \mu \mathrm{V})$.
In the second patient vitreopapillary traction had been present for 10 years. Postoperatively, optic nerve atrophy progressed and visual acuity decreased from $10 / 200$ to $4 / 200$.

In 10 of 11 patients, who were examined prospectively, a postoperative reduction of latency in VEP was observed (mean 22.25 ms; $16 \%$ lower than preoperatively, Fig 3B). In eight of those 11 patients amplitudes in VEP improved after vitrectomy (Fig 3A). Figure 4 shows an example of the PVEP in a patient who experienced a rapid postoperative reduction of latency and improvement in VEP amplitude within a week after surgery. Visual field examinations remained stable before and after vitrectomy. Although concentric constriction of the visual field due to panfundus laser photocoagulation was observed, none of the patients developed paracentral scotomas. Furthermore none of the patients showed typical glaucomatous scotomas and no correlation was found between duration of vitreopapillary traction and postoperative visual function.

\section{Discussion}

Vitreoretinal traction plays a role in a number of different diseases all due to effects at the vitreoretinal interface. ${ }^{8}$ Visual dysfunction frequently results from macular heterotopia. $^{347-13}$ In diabetic patients there are glucose induced effects upon vitreous proteins ${ }^{14}$ that result in diabetic vitreopathy, ${ }^{15}$ which in conjunction with neovascularisation into the posterior vitreous cortex ${ }^{16}$ causes circumscribed retinal detachments in areas of vitreoretinal traction. The posterior vitreous cortex is particularly adherent to the optic disc and can exert significant force which explains vitreopapillary traction. ${ }^{3}$ In proliferative diabetic vitreoretinopathy, vitreopapillary traction is most often localised to the nasal side of the optic disc. Tractional forces on nerve fibres and vessels nourishing the optic nerve head may induce optic nerve dysfunction altering visual acuity and VEP. Visual field testing does not appear to be able to detect these effects on optic nerve function, except in cases of prolonged vitreopapillary traction, such as in patient No 4. The absence of arcuate or paracentral scotoma may be due to the fact that in these patients the pathogenic mechanism appears to be opposite to the mechanism for example in glaucoma - that is, tractional versus pressure related optic neuropathy. Although optic nerve head blood flow may be reduced in patients with diabetic retinopathy as well as in patients with chronic glaucoma, functional effects on the optic nerve, like reduction of visual acuity or alterations in VEP can be present in both diseases. In patients with vitreopapillary traction affecting only the papillomacular bundle, the mechanical forces may reduce visual acuity without affecting the central visual field. Whereas in pressure related glaucoma, damage arises at the edges of the optic nerve head (Fig 5B), leading to thinning of nerve fibres and vessels followed by a reduction of perfusion. ${ }^{17}$ 

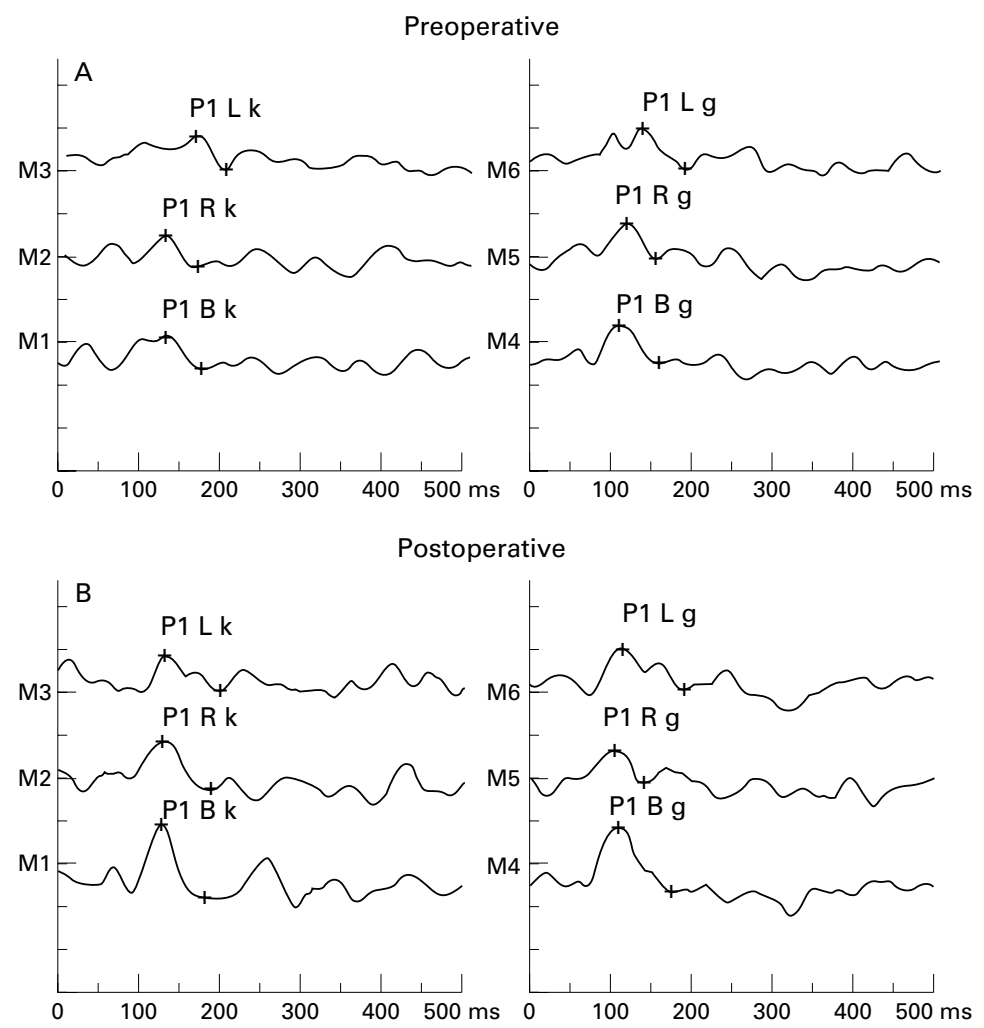

Figure 4 Pattern visual evoked potentials (PVEP) in a 50 year old patient with diabetic vitreopapillary traction in the left eye before $(A)$ and 1 week after $(B)$ vitrectomy. $(R=$ right eye, $L=$ left eye, $k=$ small pattern, $g=$ large pattern). An increase in amplitude is seen in tracings $M 1, M 3$, and $M 4$.

In diabetic vitreopapillary traction probably an elongation of optic nerve fibre bundles occurs, which consecutively leads to their thinning and a decrease of blood flow. A possible explanation for visual improvement after vitrectomy in eyes with vitreopapillary traction due to proliferative diabetic vitreoretinopathy might be that vitreoretinal traction on the optic nerve head can lead to elongation and thinning of optic nerve fibres (Fig 5A). This distortion of normal anatomy could interfere with axo-

A

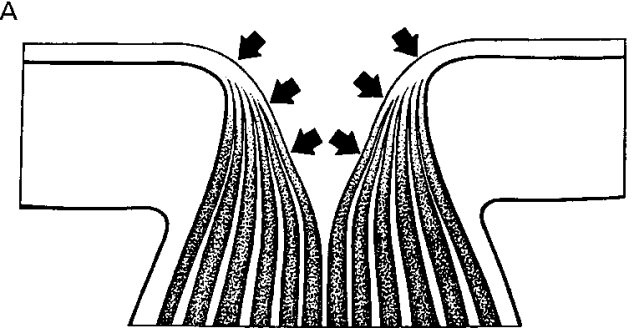

Figure 5 Schematic diagram of changes in the optic nerve head secondary to vitreopapillary traction $(A)$ and in glaucoma (B). (A) Tractional forces of the vitreous on the nerve fibres to the nasal side are responsible for elongation and thinning of these nerve fibres and their vessels, leading to a reduction of axoplasmatic flow and blood flow. This causes visual dysfunction and optic nerve head changes. (B) Typical optic nerve head damage caused by elevated intraocular pressure. Damage arises at the edges of the optic nerve head, leading to thinning of nerve fibres and vessels followed by a reduction of perfusion.

B plasmatic flow leading to a reduction of axolemmal transmission, which manifests as abnormal VEP measurements. Additionally, there might be a vascular component. Vitreopapillary traction could reduce the diameter of the blood vessels nourishing the optic nerve head, causing ischaemia of the papilla. Both mechanisms - that is, decreased axoplasmatic transport in the optic nerve fibres (neurogenic) as well as a mechanical restriction of blood flow (vasogenic) seem to be reversible in most cases. This hypothesis is supported by an increase of visual acuity, an improvement of VEP latency, and an increase in VEP amplitude in most of the patients after surgical release of vitreopapillary traction. Further studies, especially measuring optic nerve blood flow-for example, with laser Doppler flowmetry, are needed to prove the vascular hypothesis. The observed reversibility, however, is similar to the findings described in early glaucoma. ${ }^{18-20}$ It still remains unclear how duration and intensity of vitreopapillary traction contributes to the amount of optic nerve damage. Nevertheless, it can be suspected that long term traction will lead to irreversible optic neuropathy. Therefore, in eyes with vitreopapillary traction due to proliferative diabetic vitreoretinopathy, early vitrectomy should be considered as soon as visual function is reduced to avoid irreversible optic nerve atrophy. The clinical factors to follow may be visual acuity and the latency as well as amplitude of visual evoked potentials.

1 Stransky TJ. Diabetic papillopathy and proliferative retinopathy. Graefes Arch Clin Exp Ophthalmol 1986;224:46-50.

2 Slavin ML. Chronic asymptomatic ischemic optic neuropathy. A report of two cases in adults with diabetes mellitus. F Clin Neuroophthalmol 1997;7:198-201.

3 Inoue M, Tsukahara Y. Vascular optic neuropathy in diabetes mellitus. Fpn f Ophthalmol 1997;41:32-1.

4 Bustros S de, Thompson JT, Michels RG, et al. Vitrectomy for progressive proliferative diabetic retinopathy. Arch Oph-

5 Pendergast SD, Martin DF, Proia AD, et al. Removal of optic Pendergast SD, Martin DF, Proia AD, et al. Removal of optic
disc stalks during diabetic vitrectomy. Retina 1995;15:25-8. disc stalks during diabetic vitrectomy. Retina 1995;15:25-8.
Zivojnovic R. Silicone oil in vitreoretinal surgery. Dordrecht, Zivojnovic R. Silicone oil in vitreoretinal surgery.
Netherlands: Nijhoff/Junk Publishers, 1987.

7 Kroll P, Meyer-Ruesenberg HW, Busse H. Vorschlag zur Stadieneinteilung der proliferativen diabetischen Retinopathie. Fortschr Ophthalmol 1987;84:360-3.

8 Sebag J. Anatomy and pathology of the vitreo-retinal interface. Eye 1992;6:541-6.

9 Bergin DJ. Nasal heterotopia of the macula with persistent hyaloid vessel. I Pediatr Ophthalmol Strabismus 1978;15: 373-5.

10 Bottoni FG, Eggink CA, Cruysberg JRM, et al. Dominant inherited tilted disc syndrome and lacquer cracks. Eye 1990;4:504-9

11 Hegab S, Sheriff SMM. Retinal dysplasia involving the optic disc and macula. Oc Surg News Internat 1994;10:45-51.

12 Maguire AM, Green WR, Michels RG, et al. Recovery of intraocular Toxocara canis by pars plana vitrectomy. intraocular Toxocara canis by
Ophthalmology 1990;97:675-80.

13 Michael JC, Venecia G de, Bresnick GM. Macular heterotopia in proliferative diabetic retinopathy. Arch Ophthalmol 1994:112:14-9.

4 Sebag J, Buckingham B, Charles MA, et al. Biochemical abnormalities in vitreous of humans with proliferative diabetic vitreoretinopathy. Arch Ophthalmol 1992;110:1472-7.

15 Sebag J. Diabetic vitreopathy. Ophthalmology 1996;103: 205-6.

16 Faulborn J, Bowald S. Microproliferations in proliferative diabetic retinopathy and their relation to the vitreous. Graefes Arch Clin Exp Ophthalmol 1985;223:130-6.

17 Heilmann K, Richardson KT. Glaucoma, conceptions of a disease. Stuttgart: Thieme-Verlag, 1978;156-7.

18 Flammer J, Drance SM. Reversibility of glaucomatous visual field defect after acetacolamide therapy. Can $\mathcal{F} O p h-$ thalmol 1983;18:13-41.

19 Pillunat LE, Lang GK, Harris A. The visual response to increased ocular blood flow in normal pressure glaucoma. increased ocular blood flow in no

20 Sawada A, Kitazawa Y, Yamamoto T, et al. Prevention of visual field defect progression with brovincamine in eyes with normal-tension glaucoma. Ophthalmology 1996;103:283-8. 